\title{
Vorwort zum Themenheft
}

\section{Die Bahá'í-Religion}

Der Historiker Prof. Arnold Toynbee schrieb, die Bahá'í-Religion habe das Potenzial eine neue Zivilisation zu etablieren und kein Geringerer als Leo Tolstoi ging davon aus, dass Baháu'lláh, der Offenbarer der Bahá'í-Religion, den Schlüssel dazu habe, den Menschen die reinste Form religiöser Lehre nahezubringen. Der Evolutions- und Systemtheoretiker Prof. Ervin Laszlo wiederum vertritt den Standpunkt, dass die Bahá'í-Gemeinden weltweit eine positive Rolle bei der Neugestaltung der einen Welt spielen können. Eingedenk dieser und weiterer Stimmen kann die zunehmende akademische Auseinandersetzung mit einer neuen globalen Bewegung, die die Welt religiös aufzuklären versucht und dabei den Anspruch einnimmt, Glaube und Wissenschaft in Einklang zu bringen, nur einträglich sein.

Als ich im Jahr 2011 das ,Handbuch Bahá'í von Prof. Manfred Hutter in dieser Zeitschrift besprach, konnte ich mir nicht vorstellen, dass eines Tages ein Themenheft der Bahá'í-Religion gewidmet sein wird. Die Autoren, die für dieses Themenheft gewonnen werden konnten, beleuchten die Bahá'íReligion aus juristischer, religionswissenschaftlicher, islamwissenschaftlicher, sozialwissenschaftlicher, architektonischer und nicht zuletzt aus einer Bahá'íPerspektive.

Für Prof. Hoda Mahmoudi ist das Hauptziel der Bahá'í-Religion die Schaffung eines globalen Friedens durch die Anwendung spirituellen Wissens im Einklang mit wissenschaftlichen Erkenntnissen. Sie erforscht in diesem Zusammenhang die Schriften Bahá'u'lláhs und geht dabei auf den schwedischen Sozialwissenschaftler Göran Therborn ein, der die Interdependenz der Menschen weltweit hervorhebt und Migrationsströme und kulturellen Austausch infolge von Kapitalflüssen untersucht. Zudem spielt bei den sozialwissenschaftlichen Erkenntnissen von Mahmoudi die Politikwissenschaftlerin Iris Marion Young eine wichtige Rolle. Sie geht auf die Möglichkeiten einer transnationalen Zusammenarbeit auf dem Weg zu mehr Gerechtigkeit und Frieden ein. Baháu'lláhs Lehren liefern ihrer Analyse zufolge einen Plan für die Schaffung eines globalen Friedens. Das zentrale Prinzip der Offenbarungsreligion der Bahá'í sei die Einheit der Menschheit als höchste Stufe in der Entwicklung des menschlichen Lebens auf diesem Planeten. Eine solche Einheit könne durch die Errichtung eines Weltbundesstaates realisiert werden. 
Dr. Sasha Dehghani arbeitet in seinem Beitrag die Erforschung und Klassifizierung der Bahá'í-Religion in Deutschland aus einer religions- und geisteshistorischen Perspektive auf. Es gab um die Zeit des Ersten Weltkriegs bereits erste akademische Stimmen - in erster Linie aus dem Bereich der deutschsprachigen Orientalistik -, welche den Bahá'í-Glauben als eine eigenständige und universelle Religion einordneten. Doch erst nach der Katastrophe des „Dritten Reichs“, in dem die deutsche Bahá'í-Gemeinde schweren Repressalien ausgesetzt war, begann sich unter den deutschen Theologen und Religionswissenschaftlern - vor allem unter Vertretern der „Religionswissenschaft des Verstehens“ - die Einordnung des Bahá'í-Glaubens als neue Weltreligion zu etablieren. Dehghani erklärt, wie diese Anerkennung in der Nachfolgegeneration der deutschen Theologen und Religionswissenschaftler bis in die Gegenwart hinein fortgesetzt und ausgebaut worden ist.

Prof. Dr. Emanuel Towfigh setzt sich mit den theologischen Grundlagen des Bahá'í-Rechts auseinander. Ein besonderes Charakteristikum der Bahá'íReligion ist der hohe Grad rechtlicher Prägung der religiösen Lehre. Sie differenziert zwischen einer säkularen und einer sakralen Rechtssphäre und begründet auf religiöser Basis die Verpflichtung, dem staatlichen Recht grundsätzlich Folge zu leisten. Das religiöse Recht der Bahá'í-Religion postuliert damit gleichsam eine Kollisionsregel, die es sowohl dem religiösen Recht als auch der staatlichen Rechtsordnung ermöglicht, unter der Vermeidung von Konflikten den eigenen Geltungsanspruch durchzusetzen. Dieses Zusammenspiel hat in Deutschland in der Vergangenheit sowohl das religiöse Recht der Bahá'í-Religion als auch das deutsche Religionsverfassungsrecht geprägt, wie der Beitrag nachzeichnet.

Dr. Armin Eschraghi hat sich mit den Motiven des „Anti-Bahaismus“ im Iran beschäftigt und untersucht, wie sich dieser unter dem Eindruck gesellschaftlicher und politischer Entwicklungen im Laufe der vergangenen eineinhalb Jahrhunderte gewandelt hat. So werden die Bahá'í, die ursprünglich als religiös „Irregeleitete“ dargestellt wurden, im Laufe der Zeit zu Volksfeinden und Agenten fremder Mächte stilisiert. Sie wurden von den Islamisten zum politischen Feind erklärt und die herrschenden islamistischen Machthaber kolportierten, die Bahá'í seien Feinde der Nation. Diese Entwicklung lässt auch Rückschlüsse auf das wandelnde Selbstverständnis beziehungsweise die Selbstdarstellung des politischen Islam im Iran zu.

In der Miszelle beschäftigt sich Dr. Adam Robarts mit den Tempeln der Bahá'í, auch „Häuser der Andacht“ genannt. Dabei konzentriert sich Robarts auf die architektonische und symbolische Ordnung des Tempels in Wilmette, Illinois. Die Errichtung eines Tempels diene dem eigenen spirituellen Leben und sei deshalb Ausdruck der persönlichen Interaktion mit dem Göttlichen. 
Zugleich repräsentiere ein Tempel aber auch das Konzept eines Dienstes an der Menschheit. Besonders beeindruckend an dem Tempel sind die verschiedenen religiösen Symbole, die verdeutlichen, dass die religiöse Wahrheit nicht absolut sei, sondern relativ, nicht endgültig, sondern progressiv.

Die vier Rezensionen liefern Einblicke in eine Auswahl historischer und philosophischer Bahá'i-Literatur. Die tief greifenden Erkenntnisse des Religionsphilosophen Prof. Roland Faber heben die Relativität religiöser Wahrheit und religiösen Pluralismus hervor. Dr. Moojan Momen geht in seinem Werk auf die frühe Geschichte der Babí- und Bahá'í-Gemeinden ein und Guido Ettlich schildert die verschiedenen Facetten des Lebens von Konsul Albert Schwarz in Deutschland. Besonders aktuell ist die Rezension von Dr. Johannes Rosenbaum, der das Buch des Iranisten Prof. Fereydun Vahman rezensiert. In seinem Buch wird die Kontinuität und Steigerung der Verfolgung der Bahá'í im Iran in den letzten 175 Jahren beschrieben.

Ich bin nicht nur der Redaktion, der redaktionellen Geschäftsführerin der Zeitschrift, Dr. Martina Bitunjac, sowie Moritz Reininghaus und Prof. Dr. Gideon Botsch zu Dank verpflichtet, sondern vor allem Prof.Julius H. Schoeps, den Herausgeber der Zeitschrift, ohne dessen Interesse die Entstehung eines solchen Themenheftes nicht möglich gewesen wäre.

Dr. Wahied Wahdat-Hagh

Berlin, 2020 